\title{
Comment valoriser dans I'alimentation animale, les graines de soja produites en France ? Comparaison de deux procédés de transformation : I'aplatissage-cuisson-pression et l'extrusion-pression
}

\author{
Alain QUINSAC ${ }^{1}$ \\ Françoise LABALETTE ${ }^{2}$ \\ Patrick CARRÉ ${ }^{3}$ \\ Mathieu JANOWSKI ${ }^{4}$ \\ Frédéric FINE ${ }^{1}$ \\ ${ }^{1}$ CETIOM, 11 rue Monge, Parc \\ Industriel, 33650 Pessac \\ <quinsac@cetiom.fr> \\ ${ }^{2}$ ONIDOL, 11 rue de Monceau, 75008 \\ Paris \\ ${ }^{3}$ CREOL, 11 rue Monge, Parc Industriel, \\ 33650 Pessac \\ ${ }^{4}$ La Mecanique Moderne, ZAC \\ Artoipole, 62060 Arras Cedex 9
}

Article reçu le 24 septembre 2012

Accepté le 2 octobre 2012

\section{Opportunités \\ et contraintes \\ de la filière de \\ production de soja en France}

La dépendance en protéines de la France a été réduite ces dernières années par la mise sur le marché de quantités importantes de coproduits de la fabrication des biocarburants (drêches et tourteaux) et se situe nettement en dessous de la moyenne européenne (39 \% vs. $67 \%$ ) (Unip, 2012). Malgré ce progrès, près de quatre millions de tonnes de tourteau de soja sont encore

\begin{abstract}
How to develop the use for animal feeding, of soybean produced in France? Comparison of two processes: flaking-cooking-pressing and extrusionpressing

Cooking-pressing or extrusion-pressing processes applied to soybeans produce partially deoiled cake used for animal feeding. These two processes were studied and compared with a view to implementation in an industrial unit. The various factors of hydrothermal treatment for conditioning the beans were studied at different scales (batch from $2 \mathrm{~kg}$ to $100 \mathrm{~kg}$, and continuous flow at $100 \mathrm{~kg} / \mathrm{h}$ ) and the results showed the need for moist cooking to effectively deactivate the antitrypsin factors (FAT) while maintaining protein solubility. The extrusion-pressing was more effective for deoiling cakes than cookingpressing (5.4 vs. $6.7 \%$ ) but less robust for deactivation of FAT (6.5 vs. $3.8 \mathrm{TIU} / \mathrm{mg}$ ). The techno-economical study was carried out to simulate the crushing costs and net margins of both processes in a plant capacity of $20000 \mathrm{t} /$ year. It showed that the costs of both methods were similar (about $33 € / t$ ). Gross margins were evaluated between 18 and 84 $€ / t$ in three valuation assumptions based on market price of oil, the mode value of the meal and the residual oil, the level of premiums GMO traceability and transport. The cooking-pressing process can also be applied to sunflower and rapeseed and versatility is an advantage to ensure continuous operation of the crushing unit in case of supply difficulties in soybean.
\end{abstract}

Key words: soybean, extrusion, pressing, cooking, valorization, soymeal

importées chaque année. L'efficience nutritionnelle de cette matière première la positionne avantageusement dans la formulation d'aliments pour tout type d'animaux et la rend difficilement substituable dans les aliments pour les volailles, en raison de sa forte densité protéique et énergétique.

Cependant, ces tourteaux issus très majoritairement de graines de soja génétiquement modifiées (GM), ne répondent pas à l'évolution de certains cahiers des charges des productions animales de qualité qui exigent des matières premières protéiques végétales non GM. Des filières d'approvisionnement à l'import de soja non GM se sont créées, mais ne donnent pas toujours satisfaction à cause des fluctuations des cours et des garanties de qualité. De plus, elles n'établissent pas de lien au territoire, critère de plus en plus pris en compte.

Une alternative est I'utilisation du soja produit en France, dont les 100000 tonnes récoltés actuellement par an sont partagées entre l'alimentation humaine et animale. L'alimentation humaine est un secteur en pleine croissance où la valorisation de la graine est importante et conduit à une rémunération supérieure du producteur et du collecteur par le jeu de contrats spécifiques. Les volumes de graines dirigés vers l'alimentation

Pour citer cet article : Quinsac A, Labalette F, Carré P, Janowski M, Fine F. Comment valoriser dans I'alimentation animale, les graines de soja produites en France ?Comparaison de deux procédés de transformation : I'aplatissage-cuisson-pression et l'extrusion-pression. OCL 2012 ; 19 (6) : 347-357. doi : $10.1684 /$ ocl.2012.0484 
animale ne bénéficient pas, avec les modes de transformation utilisés (extrusion, toastage), d'une valorisation optimale et les produits transformés destinés à l'alimentation animale sont très fortement concurrencés par le tourteau de soja importé. Du fait de ce débouché insuffisamment rémunérateur, la compétitivité du soja par rapport aux autres cultures (maïs et blé dur en particulier) s'est trouvée réduite aux yeux des producteurs et des structures de collecte, malgré des avantages agronomiques et environnementaux certains. Cette situation a contribué fortement à détourner les surfaces de soja (actuellement environ 50000 ha en France) des débouchés en alimentation animale alors que ceux-ci sont considérables. Un retournement de tendance ne peut passer que par la transformation de la graine en produits davantage compétitifs, par des procédés adaptés au contexte de la production en France et aux besoins des utilisateurs.

Nous nous proposons de passer en revue les modes actuels de transformation du soja en France puis de décrire la cuisson-pression et l'extrusion-pression, deux procédés technologiques que nous avons étudiés en raison de leur adaptation à la taille des bassins de production du soja en France et d'une meilleure valorisation attendue de la graine.

\section{La transformation des graines de soja en France}

Les graines de soja, matière première riche en protéines (environ $34 \%$ sur la matière brute $\mathrm{MB}$ ) et en huile (18-22\% $M B)$, ne peuvent être utilisées crues pour l'alimentation des monogastriques et des jeunes ruminants car elles renferment des facteurs antinutritionnels (principalement des facteurs anti-trypsiques, FAT) qui réduisent fortement leur valeur nutritionnelle. Les FAT sont détruits efficacement par des traitements thermiques pendant la transformation de la graine: soit par les procédés d'extrusion ou de toastage appliqués à la graine entière sans déshuilage, soit par l'étape de désolvantation-toastage dans le procédé de trituration classique à la suite de l'extraction de I'huile à I'hexane. Ce dernier procédé a été appliqué sur la plus grande partie du soja produit en France jusqu'en 2003 par l'usine SAIPOL de Sète. À partir de 2003, le déclin de la culture de soja en France consécutive à la baisse des aides et à un contexte des prix agricoles défavorable à cette culture a aussitôt entraîné la réorientation de l'activité de trituration de cette usine. En effet, la trituration classique avec extraction à I'hexane n'est rentable que pour des débits importants (environ $400 \mathrm{t}$ / jour) et la récolte de soja était passée en dessous du seuil critique. Depuis, les modes de transformation de la graine de soja en France en vue d'une utilisation en alimentation animale sont presque exclusivement l'extrusion, le toastage et I'expansion-toastage, procédés qui ne modifient pas la composition de la graine en huile et protéines.

Les capacités de transformation de la graine de soja en France sont d'environ 20000 t/an pour l'extrusion, 50000 t/an pour l'expansion-toastage et 50000 t/an pour le toastage. L'enquête réalisée en 2008 dans le cadre de l'étude ONIDOLCETIOM (Labalette et al., 2010) a montré les difficultés éprouvées par les transformateurs pour utiliser leur outil à plein, le maintenir en bon état et produire des matières premières concurrentielles du tourteau de soja importé, si bien que ces outils sont devenus parfois surdimensionnés et vieillissants. Certaines unités importantes d'extrusion de soja ont d'ailleurs cessé leur activité récemment.

L'extrusion ou le toastage ont pour fonction principale de réduire l'activité des FAT par effet thermique. L'extrusion ou l'expansion permettent en outre d'améliorer la disponibilité des nutriments et donnent à la graine une valeur énergétique supérieure. Ainsi, les graines extrudées sont particulièrement indiquées pour l'alimentation de jeunes animaux. Ces modes de transformation ont cependant I'inconvénient de conserver dans la graine la totalité de la matière grasse qui sera valorisée dans la formule de l'aliment au même niveau qu'une source d'énergie substituable moins onéreuse, comme l'amidon. La présence d'un contenu élevé en huile et protéines se révèle en effet, être un handicap pour la graine de soja extrudée ou toastée, pour entrer dans des formules d'aliment à moindre coût. L'avantage nutritionnel de la graine extrudée est d'ailleurs relativisé par certains fabricants d'aliments composés qui ne la considèrent plus comme une matière première irremplaçable pour les jeunes animaux mais plutôt comme un produit bénéficiant d'une image favorable qu'il faut maintenir dans certains d'aliments. II en résulte que dans la plupart des formules, la graine de soja extrudée ou toastée est fortement concurrencée par l'association moins coûteuse, du tourteau de soja importé et d'une matière première riche en énergie moins coûteuse.

\section{Comment valoriser davantage la graine de soja?}

La valeur économique de I'huile étant nettement supérieure à celle du tourteau (1 $300 \$ / t$ vs. 668 \$/t, Rotterdam, Oilword, 2012), il est avantageux de l'extraire le plus possible du tourteau pour la valoriser séparément. Cette approche avait été adoptée par le CETIOM en 2005 pour un projet de valorisation du "Soja de Pays " dans le sud-ouest de la France sur la base des expériences mises au point aux ÉtatsUnis dans les années 1980 pour les filières tracées "identity preservation". L'étude technico-économique comprenait la mise au point des conditions optimales d'extrusion et de pressage et l'évaluation de la marge nette. L'huile était valorisée sur le marché des matières premières, les tourteaux dans l'élevage de poulets Label Rouge et les coûts de fonctionnement étaient calculés sur la base d'une unité de trituration de 20000 t/an (Quinsac et al., 2005). Les résultats étaient globalement positifs mais le procédé extrusion-pression, handicapé par son absence de polyvalence, avait constitué finalement un frein au développement du projet. Dans un contexte de surfaces de soja déclinantes, le risque était trop grand, pour cette unité de capacité relativement importante, de manquer de graines produites localement et de devoir intégrer des coûts d'approche élevés.

L'avantage d'une installation de trituration polyvalente est de pouvoir fonctionner de manière plus régulière, sur une plus longue période de l'année, et ainsi de réduire le coût de trituration à la tonne. Dans des unités industrielles de moyenne capacité, plusieurs procédés (pression à froid, double pression avec cuisson intercalée, aplatissage - cuisson - pression) sont déjà utilisés pour triturer 
indifféremment colza et tournesol. De ces procédés, seul I'Aplatissage-CuissonPression (ACP) convient au soja car il comprend, d'une part, le traitement thermique nécessaire à la désactivation des FAT et, d'autre part, une seule phase de pressage, peu coûteuse et suffisante compte tenu de la plus faible teneur en huile des graines de soja. Son adaptation doit cependant être testée avec précaution pour vérifier que dans les conditions d'une production industrielle, les performances seront suffisantes en termes de qualité et de rendement, pour un coût de fonctionnement acceptable.

Sous l'impulsion d'acteurs économiques de la zone Centre-Est de la France intéressés par l'adoption de ce procédé pour une unité de trituration de capacité adaptée à un approvisionnement et à un débouché régional, le CETIOM et I'ONIDOL ont engagé en 2010 et 2011, une étude détaillée des conditions optimales de sa mise en œuvre. Le procédé a été comparé à l'extrusion-pression dans ce même contexte, pour donner aux investisseurs tous les éléments techniques et économiques nécessaires à un choix raisonné.

\section{Les objectifs de l'étude}

À la différence du colza et du tournesol, la trituration du soja exige un traitement thermique adapté pour désactiver les FAT sans dégrader la digestibilité des acides aminés. Ce traitement est le point critique du process car il conditionne fortement la qualité nutritionnelle des tourteaux produits et leur valorisation. D'autre part, les performances de l'étape de déshuilage sont primordiales pour le bilan massique de la trituration et la rentabilité économique de l'opération. En conséquence, les critères d'évaluation du procédé suivants ont été retenus pour l'étude : la désactivation des FAT, la conservation de la solubilité des protéines et de leur digestibilité, le rendement d'extraction de I'huile, la qualité de l'huile et enfin, la performance économique de l'unité de transformation envisagée.

\section{Les propriétés des FAT}

Les FAT sont des composés protéiques présents dans les graines de légumineuses qui se fixent sur les protéases digestives et inhibent leur activité. Deux types sont présents dans la graine de soja : le facteur de Künitz, majoritaire, qui inhibe principalement la trypsine $(1,4 \mathrm{~mol} / \mathrm{mol})$ et peu la chymotrypsine $(0,1 \mathrm{~mol} / \mathrm{mol})$ et le facteur de Bowman Birk qui agit autant avec la trypsine que la chymotrypsine (2 mol$/ \mathrm{mol}$ ) (Weder et King, 1998). Ces deux inhibiteurs sont thermolabiles et selon les auteurs, l'activité antitrypsique résiduelle après un traitement thermique est attribuée au facteur de Bowman Birk (Melcion et Van der Poel, 1993) ou de Künitz (Keshun, 1997). Un effet secondaire de I'action des FAT qui contribue à augmenter leurs propriétés antinutritionnelles, est la synthèse accrue par le pancréas de trypsine entraînant une forte consommation d'acides aminés soufrés qui deviennent alors insuffisants pour les autres besoins de l'organisme.

\section{La réduction des FAT : extrusion sèche ou humide, cuisson, traitements à la vapeur, toastage?}

Les méthodes de traitement publiées pour la réduction des FAT sont assez nombreuses et empruntent la plupart des techniques connues de chauffage (autoclave, conduction, trempage et ébullition, micro-ondes, extrusion sèche ou humide, infrarouge, etc.). L'étude du procédé extrusion-pression (Quinsac et al., 2005) a montré qu'il est possible par l'extrusion sèche de réduire les FAT d'un soja initialement à 40-45 UTI/mg de graines jusqu'à une valeur proche de $5 \mathrm{UTI} / \mathrm{mg}$ de tourteau gras à condition que la température dans le fourreau de l'extrudeur soit de $150{ }^{\circ} \mathrm{C}$. Les tourteaux avaient par ailleurs une teneur en huile résiduelle inférieure à $5 \%$ et une valeur de solubilité des protéines de $70 \%$ dans la soude. En extrusion humide et en raison de l'effet de la vapeur, la température nécessaire peut être sensiblement réduite. L'inconvénient majeur de cette technique dans un schéma extrusion-pression est I'humidification importante de l'extrudat défavorable à l'efficacité du déshuilage des graines par le pressage. L'extrusion humide pénalise donc soit le bilan énergétique du process si on procède à un séchage, soit le bilan matière avec moins d'huile extraite si I’humidité est conservée.

Les traitements de cuisson pour être efficaces doivent faire appel à certains niveaux de température et d'injection de vapeur mais les durées de traitement nettement plus élevées que dans un extrudeur, font que ces niveaux restent raisonnables. Ainsi les températures varient entre 95 et $105^{\circ} \mathrm{C}$, et la durée de traitement entre 40 et $60 \mathrm{~min}$ selon Rackis et al. (1974) et Hekelman et al. (1992). Le toastage à des températures plus élevées $\left(120^{\circ} \mathrm{C}\right.$ ou $\left.135^{\circ} \mathrm{C}\right)$ réduit fortement le temps d'exposition nécessaire (respectivement 7,0 ou 4,5 $\mathrm{min}$ ) et augmente le risque d'une sur-cuisson.

En conclusion, les traitements par extrusion et cuisson pour réduire les FAT sont possibles et d'autant plus efficaces que I'humidité du milieu est élevée. Les températures de traitement peuvent ainsi être abaissées (vers $120{ }^{\circ} \mathrm{C}$ avec l'extrusion et $100{ }^{\circ} \mathrm{C}$ avec la cuisson). Cet effet devra cependant être limité car I'humidité est préjudiciable à la performance du pressage consécutif à cette étape et pénalise le bilan massique de la transformation en réduisant la part d'huile extraite. La désactivation des FAT par traitement thermique a une incidence directe sur la qualité des protéines et il est nécessaire de contrôler le degré de cuisson pour sauvegarder leur digestibilité. Plusieurs tests directs ou indirects permettent d'évaluer I'intensité du traitement appliqué et son adéquation au but recherché (tableau 1).

L'activité des FAT est déterminée par la méthode dédiée (AOCS-Ba 12-75), mais souvent dans les usines, des contrôles plus rapides sont réalisés par des méthodes indirectes qui mesurent la sur-cuisson (test au rouge de crésol), la sous-cuisson (test de l'uréase) ou la modification de la solubilité des protéines (solubilité dans $\mathrm{KOH}$ ou indice PDI).

\section{Les essais expérimentaux}

Les essais pour l'étude du procédé basé sur la cuisson-pression ont été réalisés en trois étapes :

1) Atelier CREOL (Pessac): tests de cuisson à l'échelle du kg pour modéliser l'effet des traitements hydrothermiques à l'aide d'un mini-cuiseur instrumenté (figure 1) permettant de contrôler précisément la température, I'injection de vapeur et la durée du traitement, selon un plan d'expériences ; 
Tableau 1. Comparaison des différents tests et valeurs attendues selon le traitement thermique (selon Lareal, 2009)

\begin{tabular}{|llllll|}
\hline $\begin{array}{l}\text { Traitement } \\
\text { sur soja }\end{array}$ & $\begin{array}{l}\text { FAT } \\
(\mathbf{U T I} / \mathbf{m g})\end{array}$ & $\begin{array}{l}\text { Solubilité KOH } \\
(\mathbf{\%})\end{array}$ & $\begin{array}{l}\text { Uréase } \\
(\Delta \mathbf{p H})\end{array}$ & $\begin{array}{l}\text { RDI } \\
(\mathbf{m g} / \mathbf{g})\end{array}$ \\
\hline Aucun & 25 à 60 & $>90$ & 2 & - & $\begin{array}{l}\text { PDI } \\
(\mathbf{\%})\end{array}$ \\
\hline Sous cuisson & 7 à 15 & $>85$ & 0,1 à 0,5 & $<5$ & 30 à 70 \\
\hline Cuisson correcte & 2 à 7 & 75 à 85 & $<0,1$ & 5,0 à 6,5 & $>6,5$ \\
\hline Cuisson excessive & $<2$ & $<70$ & 0 & 15 à 30 \\
\hline
\end{tabular}

2) Atelier La Mécanique Moderne (Arras): cuisson à l'échelle pilote (100 kg) en mode "batch " dans un cuiseur de dimensions $80 \times 150 \mathrm{~cm}$ avec système de brassage. Pressage par presse à vis La Mécanique Moderne, MBU 20 de capacité $100 \mathrm{~kg} / \mathrm{h}$;

3) Atelier CREOL (Pessac): cuissonpression à l'échelle pilote $(100 \mathrm{~kg} / \mathrm{h})$ en continu pour valider les conditions établies en "batch " et évaluer les performances de déshuilage. Les essais d'extrusion-pression ont été réalisés à l'échelle pilote sur les mêmes lots de graines pour bien évaluer les différences de performances entre les deux procédés. Les équipements utilisés au pilote sont un cuiseur horizontal biétagé (La Mécanique Moderne) $900 \mathrm{~mm}$ de diamètre, $2500 \mathrm{~mm}$ de longueur avec injection de vapeur, un extrudeur (France extrusion FEX1) de capacité

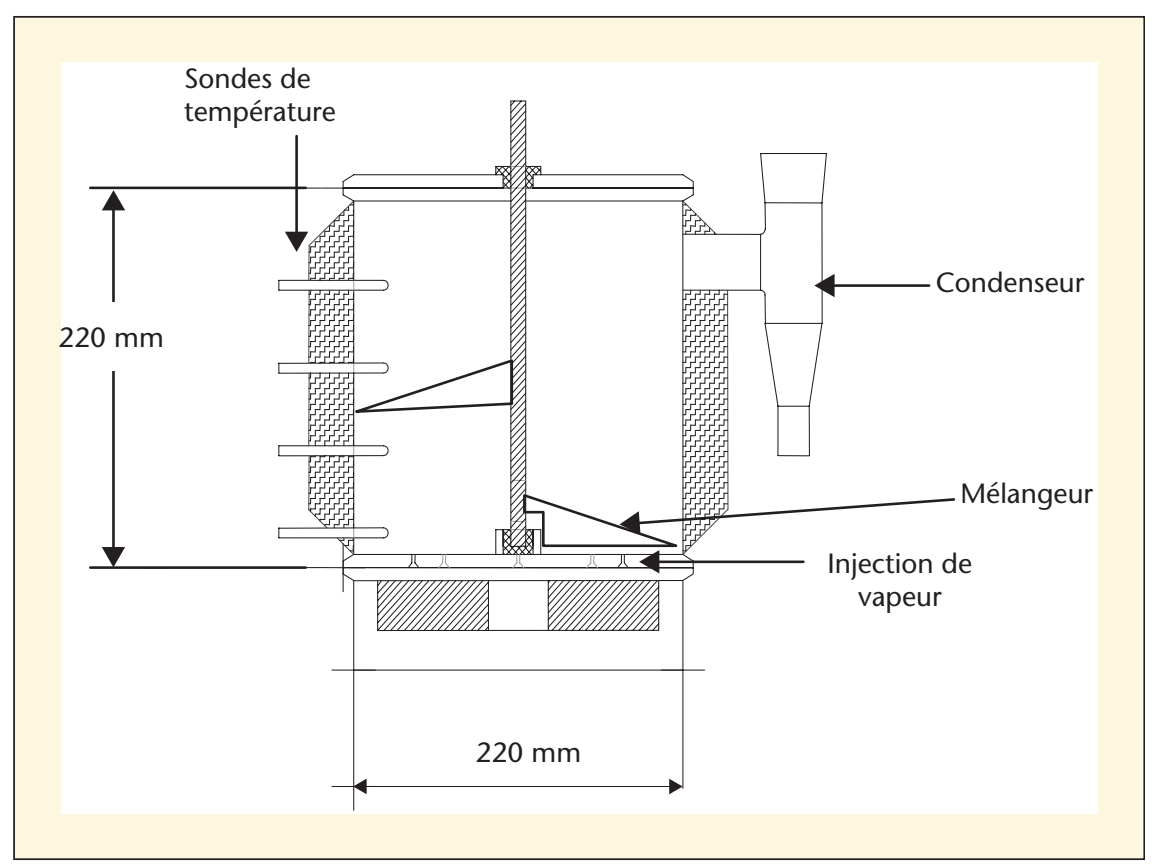

Figure 1. Représentation schématique du mini-cuiseur (capacité $2 \mathrm{~kg}$ ) (l'injection de vapeur est réalisée par des orifices situés à la base du mini-cuiseur).

$50-300 \mathrm{~kg} / \mathrm{h}$, une presse à vis (La Mécanique Moderne, MBU 75) de capacité $50-500 \mathrm{~kg} / \mathrm{h} \quad(150 \mathrm{~mm}$ de diamètre, arbre de $1800 \mathrm{~mm}$ de longueur).

La désactivation des FAT a été mesurée par la méthode directe (AOCS-Ba 1275), la qualité des protéines par la mesure de la solubilité dans la potasse, et le rendement de déshuilage, par la mesure de l'huile résiduelle (ISO 734-2) dans les tourteaux.

Les essais expérimentaux dans ces trois étapes ont été réalisés avec des lots de graines différenciés par la teneur en FAT, le lieu de production, la date de récolte et si possible, la variété. Ont été testés : un lot de la variété Isidor récolté en France en 2009 avec une teneur en FAT forte (environ $50 \mathrm{UTI} / \mathrm{mg}$ ), deux lots des variétés Hilario et Aires récoltés en Italie

350 OCL VOL. 19 N 6 novembre-décembre 2012 en 2009 avec des teneurs faibles en FAT (< $25 \mathrm{UTI} / \mathrm{mg}$ ), un lot de la variété Ascasubi récolté en France en 2010 (lot $Z)$ avec une teneur faible en FAT $(<20$ UTI mg), deux lots de graines banalisées (lots A et B) récoltés en 2009 et 2010 par des coopératives du Centre-Est de la France (région Rhône-Alpes) et de teneurs en FAT voisines de $35 \mathrm{UTI} / \mathrm{mg}$. Dans l'étude, les lots de graines de teneur initiale inférieure ou supérieure à $30 \mathrm{UTI} / \mathrm{mg}$ ont été dénommés respectivement FAT $(-)$ ou FAT (+).

\section{Résultats}

\section{Essais sur mini-cuiseur}

Des tests préliminaires réalisés à l'aide du mini-cuiseur ayant montré que la chaleur sèche à $100{ }^{\circ} \mathrm{C}$ était insuffisante pour réduire les FAT à un niveau suffisamment bas même avec une durée du traitement de $80 \mathrm{~min}$, des essais d'injection de vapeur ont été intégrés au plan d'expériences. Ces essais ont donné des résultats satisfaisants avec des niveaux résiduels en FAT inférieurs à $10 \mathrm{UTI} / \mathrm{mg}$ et ont montré les effets principaux des facteurs étudiés (tableau 2).

Les effets qui ont le plus de conséquences sur le niveau résiduel de FAT, sont dans l'ordre décroissant : I'effet variété, la durée de l'injection de vapeur (DIV), la préparation mécanique (PM), et la durée de séchage (DS). Les facteurs température de séchage (TS) et débit de vapeur (dV) peuvent être considérés comme peu significatifs. Concernant les effets sur le niveau résiduel de la solubilité des protéines (SoIP), I'ordre décroissant est le suivant: durée de I'injection de vapeur, durée de séchage, niveau en FAT initial. La préparation mécanique et le débit de vapeur ont également des effets significatifs quoique moindres.

L'analyse de variance a été réalisée en introduisant les données numériques 
Tableau 2. Calcul des contrastes (différences entre moyennes des effets des modalités (+) et (-) du plan d'expériences réalisées à l'aide du mini-cuiseur).

\begin{tabular}{|llllll|}
\hline Facteur & Valeur $(-)$ & Valeur (+) & $\begin{array}{l}\text { Niveau résiduel } \\
\text { FAT } \\
\text { (UTI/mg) }\end{array}$ & $\begin{array}{l}\text { Solubilité } \\
\text { des protéines (SolP) } \\
(\%)\end{array}$ & $\begin{array}{l}\text { Nombre } \\
\text { d'essais (1) }\end{array}$ \\
\hline Niveau initial en FAT (UTI/mg) & 21 & 47 & 2,58 & $-3,50$ & 8 \\
\hline Préparation mécanique (PM) & Flocons & Broyé & $-0,50$ & $-2,50$ & 8 \\
\hline Durée injection de vapeur (DIV) & $15 \mathrm{~min}$ & $30 \mathrm{~min}$ & $-1,58$ & $-4,88$ & 8 \\
\hline Débit de vapeur (dV) & $200 \mathrm{~g} / \mathrm{h}$ & $400 \mathrm{~g} / \mathrm{h}$ & $-0,23$ & $-2,63$ & 8 \\
\hline Température de séchage (TS) & $105^{\circ} \mathrm{C}$ & $115{ }^{\circ} \mathrm{C}$ & $-0,26$ & $-3,75$ & $-4,00$ \\
\hline Durée de séchage (DS) (2) & $\rightarrow 6 \%$ d'eau & $\rightarrow 4 \%$ d'eau & $-0,46$ & & 8 \\
\hline
\end{tabular}

(1) Le nombre d'essais se réfère aux situations comparables disponibles.

(2) Les durées de séchage correspondent aux temps déterminés par l'atteinte de la teneur en eau cible.

des paramètres et en limitant le nombre de classes pour réduire le risque de biais, possible par le caractère déséquilibré du dispositif. Pour la construction des modèles, seules les données issues du lot de graines $\mathrm{FAT}(+)$ ont été prises en compte car avec le lot FAT(-) le domaine des conditions opératoires exploré était trop restreint pour mettre en évidence les effets des facteurs censés réduire le niveau des FAT et la solubilité des protéines.

\section{Activité antitrypsique}

Dans le modèle ci-dessous, la durée d'injection de vapeur (DIV), la température de séchage (TS) et la durée du séchage (DS) expliquent $84 \%$ de la variance. Ajouter le facteur débit d'injection de vapeur n'apporte pas un gain significatif.

Graines FAT (+) FAT $=16,2-0,065$ DIV 0,075 TS $-0,045$ DS $\left(R^{2}=0,84\right)$

On remarque l'importance du facteur "durée d'injection de vapeur » (DIV) par rapport à la durée du séchage (DS), indiquant que la désactivation des FAT est favorisée par une chaleur humide.

\section{Solubilité des protéines}

Dans le modèle ci-dessous, la durée d'injection de vapeur (DIV), la température de séchage (TS) et la durée du séchage (DS) expliquent $90 \%$ de la variance. Le poids du facteur DIV est moins important que pour le modèle précédent. Les facteurs température et durées de séchage (TS et DS) ont renforcé leurs effets.

Graines FAT $(+)$ SolP $=147,6-0,196$ DIV - 0,477 TS - 0,182 DS $\left(R^{2}=0,90\right)$

En conclusion de ces essais sur minicuiseur, il apparaît que la réduction de la teneur en FAT est obtenue principalement avec le facteur " durée d'injection de vapeur » alors que pour la réduction de la solubilité des protéines, les facteurs "durée de séchage " et "température de séchage " ont aussi des effets importants. Les FAT sont plus sensibles à la chaleur humide qu'à la cuisson sèche tandis que la solubilité des protéines n'est pas sensiblement impactée par les conditions d'humidité. Donc, pour inactiver les FAT sans perdre beaucoup de solubilité, une étape de cuisson humide doit être privilégiée.

Bien que les données expérimentales obtenues avec les lots de graines FAT (-) n'aient pas permis de construire un modèle précis $(R=0,51)$, l'effet du facteur "teneur initiale en FAT " sur la teneur résiduelle en FAT et sur la solubilité des protéines a été observé.

\section{Essais de cuisson en batch (pilote de La Mécanique Moderne, Arras)}

Cinq essais ont été effectués sur les lots de graines "Isidor » en faisant varier la température de cuisson et la quantité d'eau ajoutée, pour évaluer l'effet de ces facteurs sur la cinétique de réduction des FAT (figure 2).

Les essais 1 et 2 ont été réalisées dans des conditions qui n'ont pas permis $\mathrm{d}^{\prime}$ atteindre les objectifs de température et humidité dans le cuiseur. Seuls les résultats de l'essai 1 sont reportés et montrent qu'une cuisson de $2 \mathrm{~h}$ a été nécessaire pour atteindre un niveau de FAT voisin de $10 \mathrm{UTI} / \mathrm{mg}$ de matière sèche délipidée (MSD). Les essais 3 à 5 au cours desquels un ajout d'eau a été effectué en début de cuisson, ont conduit à des niveaux de température et d'humidité favorables à la réduction des FAT. L'essai 4 réalisé en conditions sèches s'est avéré peu performant. Dans tous les essais, le niveau résiduel de solubilité des protéines est resté supérieur à $80 \%$ quelles que soient les conditions (figure 3).

Ces essais de cuisson en batch ont montré la possibilité de réduire le niveau des FAT dans un cuiseur standard sans injection de vapeur à condition de pouvoir générer des conditions de cuisson humide par ajout d'eau sur des graines chaudes. Cette cuisson humide est indispensable à la réduction du niveau de FAT à moins de $10 \mathrm{UTI} / \mathrm{mg}$ MSD. La durée nécessaire de traitement est d'environ 45-60 min (essai 3).

\section{Essais de cuisson - pression en continu au pilote CREOL}

Les essais ont été réalisés sur deux lots (A et $\mathrm{B}$ ) de graines à forte teneur en FAT récoltées en région Rhône-Alpes respectivement en 2009 et 2010, de variété courante non identifiée, et sur un lot ( $Z$ ) à faible teneur en FAT récoltées en 2010 (variété Ascasubi). Chaque lot a fait l'objet de deux essais de traitement de cuisson et pression en mode continu au pilote CREOL (tableau 3). Le cuiseur horizontal bi-étagé était utilisé en mode cuisson humide dans le premier étage et en mode séchage dans le second. Cet appareil muni d'un dispositif de mélange générant une dispersion importante des temps de séjour (avec un stock de $200 \mathrm{~kg}$ pour un débit de $200 \mathrm{~kg} / \mathrm{h}, 66 \%$ de la matière sort entre 30 et $137 \mathrm{~min}$ ), les durées de traitements testées dans les essais en mode batch ont été allongées et fixées à environ $80-90 \mathrm{~min}$. Les produits en sortie cuiseur et presse sont appelés 


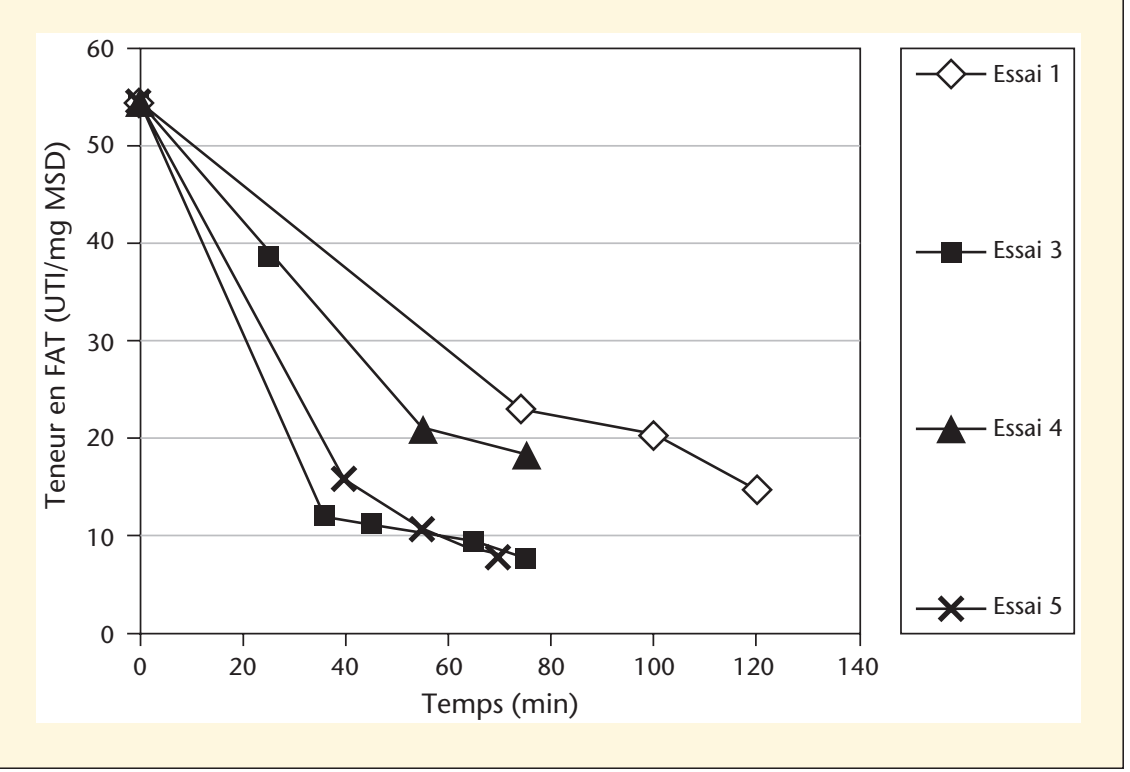

Figure 2. Cinétiques de réduction des teneurs en FAT observées dans les graines "Isidor " dans les essais de cuisson en batch $n^{\circ} 1,3,4$ et 5 .

respectivement flocons et écailles de pression.

Les conditions appliquées ont été assez voisines pour tous les sous-lots, excepté pour A1 et Z1 dont les temps de séjour dans le cuiseur sont nettement inférieurs. Ces deux sous-lots permettent d'évaluer la relation entre le temps de cuisson, la réduction des FAT et de la solubilité des protéines (figures 4 et 5 ) pour des lots de graines à teneur en FAT variant de 30 (lot Z) à $54 \mathrm{UTI} / \mathrm{mg}$ (lot A). La cuisson a été quantifiée en termes de degré Celsius $\mathrm{x}$ heure $\left({ }^{\circ} \mathrm{C} . \mathrm{h}\right)$ en multipliant la température de sortie mesurée sur les flocons par la durée de la cuisson. Une cuisson de $128 \pm 8^{\circ} \mathrm{C}$.h provoque la

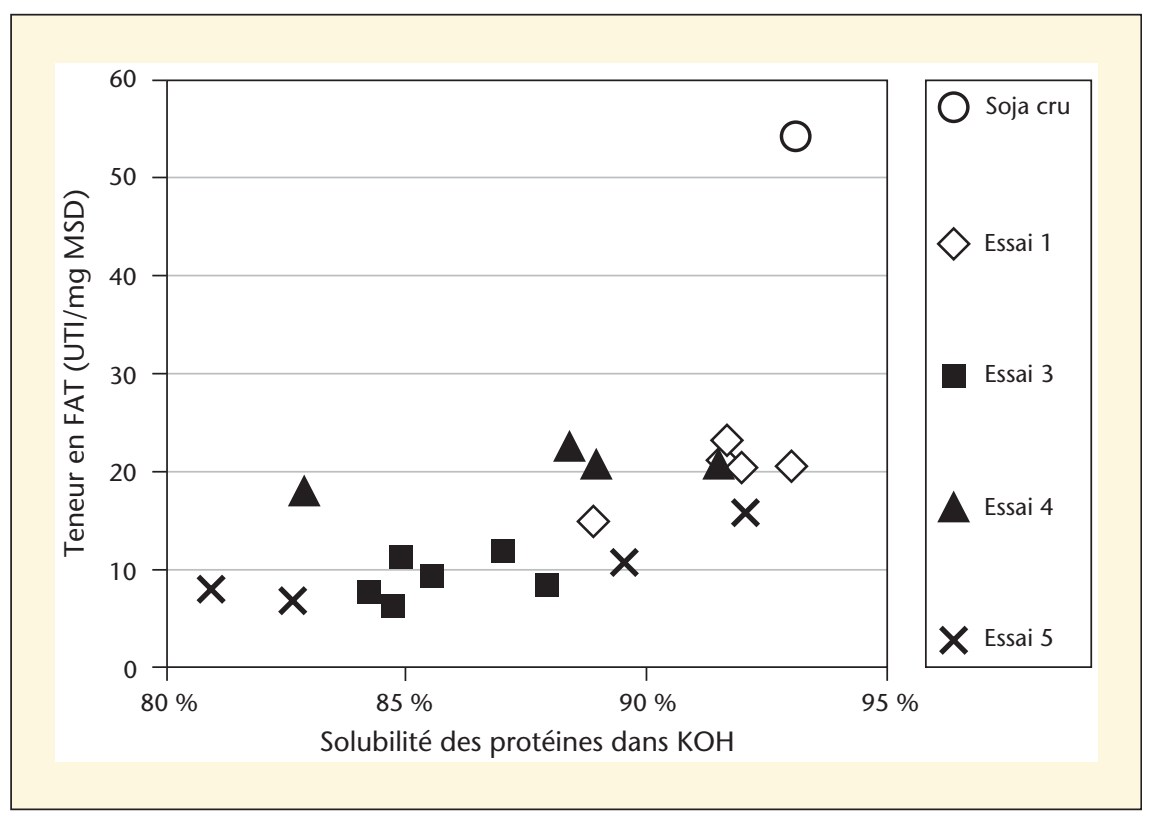

Figure 3. Niveaux résiduels de FAT et de solubilité des protéines mesurés avec les graines "Isidor" dans les essais de cuisson en batch $n^{\circ} 1,3,4$ et 5 . réduction de $90 \%$ de la teneur initiale en FAT et de $20 \%$ de la solubilité des protéines des lots $A$ et $B$. Une réduction de $95 \%$ des FAT du lot $Z$ est obtenue avec une cuisson de seulement $100^{\circ} \mathrm{C}$.h.

L'essai a montré qu'il était possible, sur des graines de teneur en FAT de 45 et $55 \mathrm{UTI} / \mathrm{mg}$ MSD (32 et $39 \mathrm{UTI} / \mathrm{mg}$ brut), de réduire le niveau en FAT à une valeur très faible inférieure à $5 \mathrm{UTI} / \mathrm{mg}$ MSD (3,5 UTI/mg brut) compatible avec une utilisation sans risque en alimentation des monogastriques, en évitant une trop grande diminution de la solubilité des protéines (de $90 \%$ à $75 \%$ ). Pour des graines à teneur initiale plus faible en FAT, les conditions $d$ 'une cuisson optimale seront encore plus faciles à obtenir. En raison du traitement de séchage dans le deuxième étage du cuiseur, les flocons cuits ont perdu environ 4 à 6 points d'humidité. Cette perte d'humidité est favorable au déshuilage et on constate comme attendu, que le lot A2 plus sec que le lot A1 (humidité : 5,5 vs. $6,8 \%)$ a conduit à des écailles de pression mieux déshuilées (huile : 7,3 vs. 9,6 \%) (tableau 3).

Les essais sur mini-cuiseur ont permis d'établir des modèles de réduction des FAT et de la solubilité des protéines en fonction des facteurs appliqués. Les essais en pilote batch ont montré que la cuisson humide était possible sans injection de vapeur avec seulement une addition d'eau sur les graines chaudes. Enfin, les essais en pilote en continu ont souligné l'importance de la dispersion des temps de séjour des graines dans les cuiseurs comportant peu d'étages et la nécessité de corriger à la hausse les durées de traitement pour s'assurer de la désactivation suffisante des FAT.

\section{Essais d'extrusion-pression au pilote CREOL}

Des conditions d'extrusion validées lors d'études précédentes sur la graine de soja (Quinsac et al., 2005) ont été appliquées de manière homogène sur les trois lots de graines (tableau 4). L'extrusion est réalisée à environ $150{ }^{\circ} \mathrm{C}$ en conditions sèches pour réduire autant que possible la teneur en eau à I'entrée de la presse. Avec ces conditions, la désactivation des FAT dans les écailles de pression pour le lot A s'est avérée insuffisante (niveau voisin de 10 $\mathrm{UTI} / \mathrm{mg}$ ), alors que les résultats sont 
Tableau 3. Conditions de cuisson des lots $A, B$ et $Z$ et caractéristiques des flocons et écailles de pression obtenues au pilote CREOL avec le procédé Aplatissage-Cuisson-Pression.

\begin{tabular}{|c|c|c|c|c|c|c|c|}
\hline & Lot & $\mathbf{A}$ & & B & & $\mathbf{Z}$ & \\
\hline \multirow{4}{*}{ Graines } & Humidité (\%) & 11,0 & & 12,7 & & 12,4 & \\
\hline & Teneur en huile (\% MS) & 22,2 & & 21,5 & & 22,2 & \\
\hline & Teneur en FAT (UTI/mg MSD) (1) & 46,5 & & 54,1 & & 30,1 & \\
\hline & Solubilité protéines $\mathrm{KOH}(\%)$ & 89 & & 95 & & 91 & \\
\hline \multirow{7}{*}{$\begin{array}{l}\text { Flocons } \\
\text { (sortie cuiseur) }\end{array}$} & Sous-lot & A1 & $\mathrm{A} 2$ & B1 & B2 & Z1 & $\mathrm{Z2}$ \\
\hline & Température cuisson $\left({ }^{\circ} \mathrm{C}\right)$ & 99 & 104 & 101 & 101 & 102 & 104 \\
\hline & Temps de séjour (min) & 21 & 78 & 91 & 86 & 59 & 92 \\
\hline & Débit $(\mathrm{kg} / \mathrm{h})$ & 202 & 171 & 176 & 173 & 196 & 175 \\
\hline & Humidité (\%) & 6,8 & 5,5 & 7,3 & 7,0 & 6,4 & 6,5 \\
\hline & Solubilité protéines $\mathrm{KOH}(\%)$ & 89,0 & 75,0 & 73,0 & 74,0 & 75 & 73 \\
\hline & Teneur en FAT (UTI/mg MSD) & 9,5 & 5,0 & 3,7 & 3,9 & 1,4 & 1,2 \\
\hline \multirow{4}{*}{$\begin{array}{l}\text { Ecailles de pression } \\
\text { (sortie presse) }\end{array}$} & Humidité (\%) & 7,0 & 5,4 & 7,1 & 7,6 & 6,3 & 7,0 \\
\hline & Teneur en huile (\% MS) & 9,6 & 7,3 & 6,9 & 7,3 & 7,0 & 7,4 \\
\hline & Solubilité protéines $\mathrm{KOH}(\%)$ & 90 & 74 & 72 & 73 & 74 & 72 \\
\hline & Teneur en FAT (UTI/mg MSD) & 9,5 & 5,2 & 3,7 & 4,3 & 1,3 & 1,0 \\
\hline
\end{tabular}

(1) MSD : matière sèche délipidée

satisfaisants pour le lot B dont la teneur initiale en FAT est supérieure. Les résultats pour le lot $Z$ sont conformes aux objectifs. Les résultats obtenus montrent qu'une teneur en eau entrée presse de l'ordre de 6,5\% permet un déshuilage satisfaisant tout en améliorant I'inactivation des FAT. II est donc

préférable de traiter des graines à 12,5 \% d'eau qu'à 11,0 \%.

\section{Comparaison des traitements de cuisson et extrusion}

Bien que les niveaux de solubilité des protéines soient assez voisins avec les

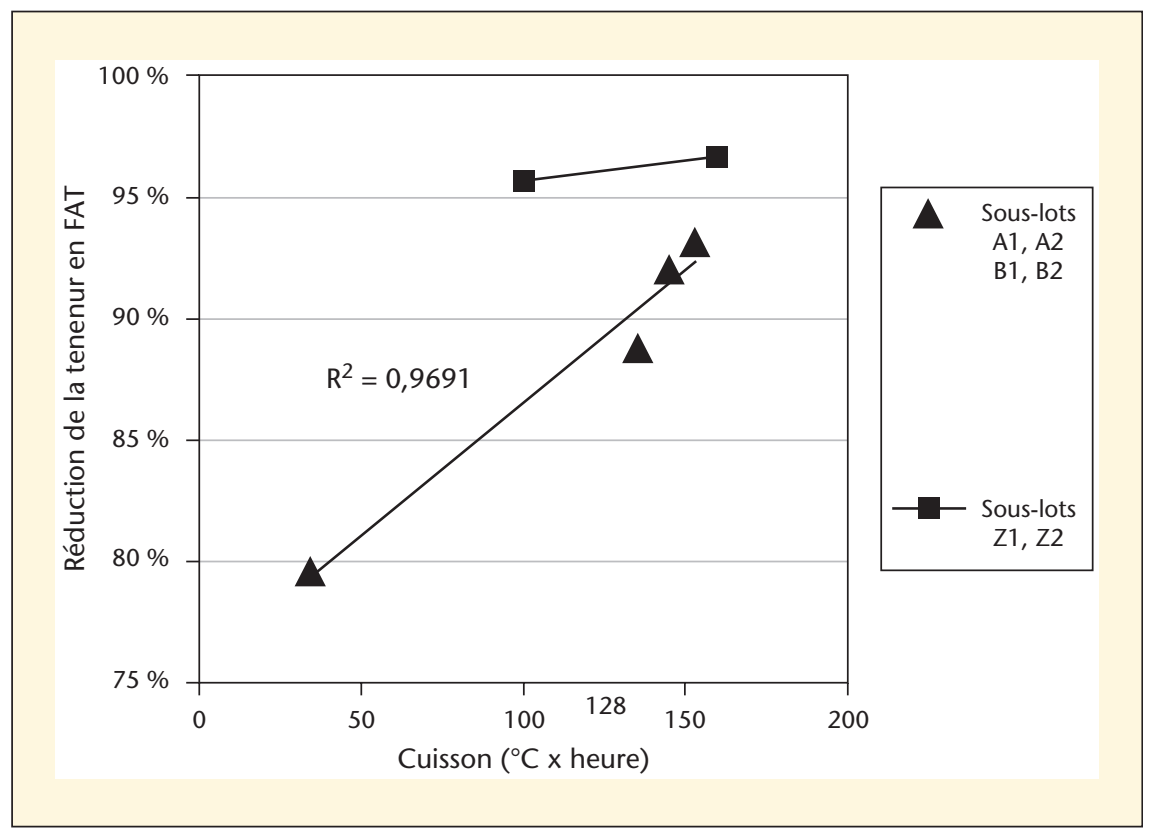

Figure 4. Effet de la cuisson sur la réduction de la teneur en FAT dans les écailles de pression préparées à partir des graines de soja FAT+ (lots $A$ et B) et FAT- (lot Z). Réduction = (teneur initiale - teneur finale)/teneur initiale). deux traitements, les niveaux de FAT après extrusion sont sensiblement plus élevés que ceux obtenus après cuisson (tableau 5). L'extrusion sèche se révèle moins efficace pour désactiver les FAT que le traitement de cuisson humide, ce qui confirme les observations faites dans l'étude sur mini-cuiseur.

En revanche, l'extrusion facilite le pressage et le déshuilage obtenu est nettement meilleur. En moyenne, les écailles d'extrusion-pression contiennent environ 1,3 point $d^{\prime}$ huile en moins que celles de cuisson-pression ce qui a un fort impact sur la valorisation des produits, aussi bien sur la quantité d'huile produite que sur la qualité du tourteau.

\section{Quel procédé pour une unité de trituration de 20000 t/an?}

La prédiction de la composition des tourteaux produits dans une usine triturant 20000 tonnes de graines par an a été réalisée sur la base des résultats des essais technologiques, aussi bien sur les valeurs moyennes que sur les variations mesurées. Les circonstances des essais réalisés chez CREOL ont été analysées pour prévoir au niveau industriel une dégradation ou au contraire et selon le cas, une amélioration des performances. L'expertise de l'équipementier La Mécanique Moderne en matière de cuisson et déshuilage à l'échelle indus- 


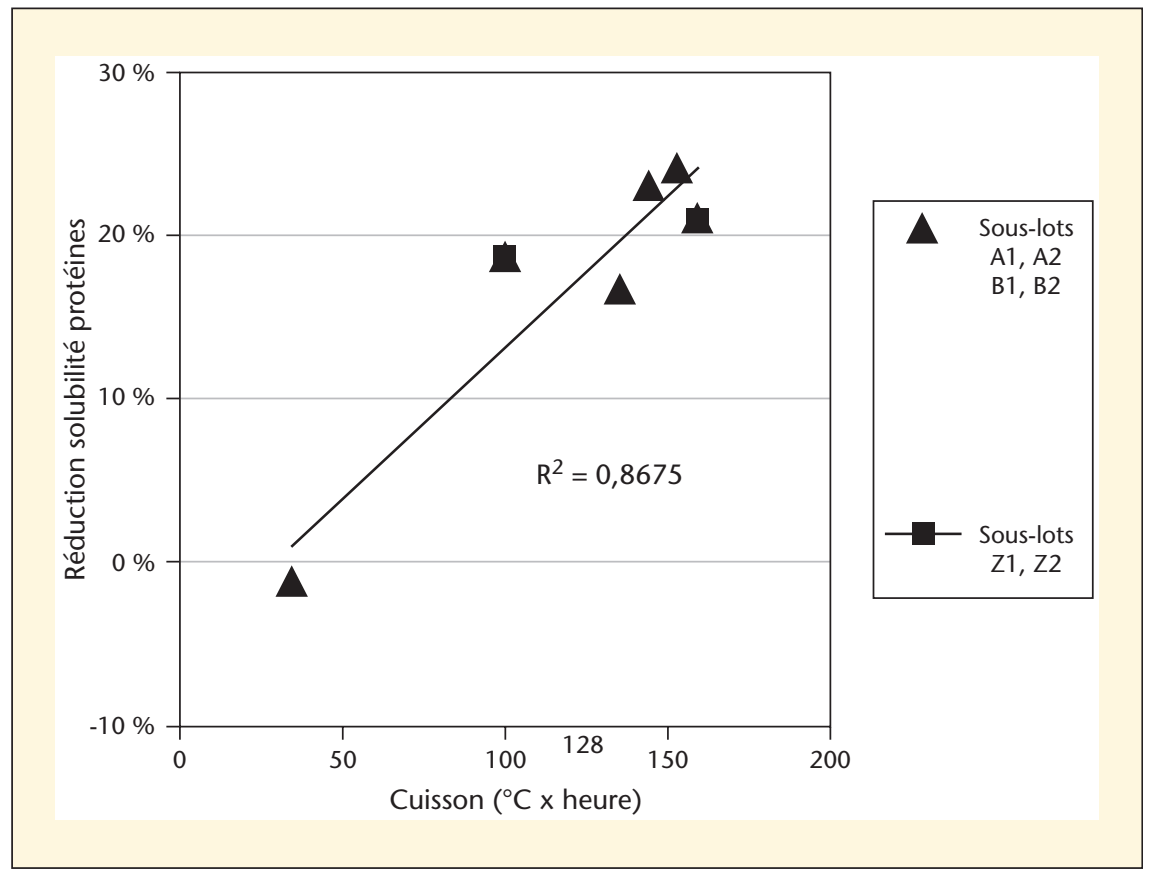

Figure 5. Effet de la cuisson sur la réduction de la solubilité des protéines dans les écailles de pression préparées à partir des lots de graines de soja FAT+ (lots A et B) et FAT- (lot Z). Réduction $=($ teneur initiale - teneur finale $) /$ teneur initiale $)$.

trielle, a été prise en compte pour définir les niveaux $d$ 'humidité et de matière grasse résiduelle dans les tourteaux.

Cette analyse (tableau 6) prédit que le procédé EP ne pourra probablement pas assurer régulièrement un niveau de FAT $<10 \mathrm{UTI} / \mathrm{mg}$ brut, contrairement au procédé ACP. Pendant les essais chez CREOL, l'extrusion sèche avait été mise en œuvre à la limite de ses possibilités, et une amélioration des performances de désactivation des FAT ne peut être espérée à l'échelle industrielle. L'extrusion humide, procédé utilisé dans les usines en activité dans la région RhôneAlpes, permet une meilleure désactivation des FAT mais parce qu'elle est inadaptée à l'opération de pressage réalisée en suivant, elle n'a pas été testée dans cette étude. En effet, les extrudats produits seraient trop humides pour être pressés efficacement, leur séchage avant pression dégraderait la qualité de I'huile et le coût énergétique du procédé serait augmenté. II serait cependant envisageable d'étudier l'extrusion à sec de graines plus humides (13-14\% d'humidité) pour vérifier l'effet positif de cette humidité sur la désactivation des FAT.

\section{Étude technico-économique}

Les données sur le déshuilage des graines (huile résiduelle des tourteaux) ont été utilisées pour alimenter un modèle de simulation technico-économique donnant des évaluations des marges brutes et nettes de trituration en fonction des hypothèses optimistes et prudentes sur le procédé (tableau 6) et d'hypothèses de valorisation des produits de la trituration (huile et tourteaux). Le bilan matière (tableau 7) montre l'intérêt du procédé EP pour une production plus importante d'huile. L'écart entre les marges brutes

Tableau 4. Conditions d'extrusion des lots A, B et Z et caractéristiques des extrudats et des écailles de pression obtenues au pilote CREOL avec le procédé Extrusion-Pression.

\begin{tabular}{|c|c|c|c|c|c|c|c|}
\hline & Lot & $\mathbf{A}$ & & B & & $\mathbf{Z}$ & \\
\hline \multirow{4}{*}{ Graines } & Humidité (\%) & 11,0 & & 12,7 & & 12,4 & \\
\hline & Teneur en huile (\% MS) & 22,2 & & 21,5 & & 22,2 & \\
\hline & Teneur en FAT (UTI/mg MSD)(1) & 46,5 & & 54,1 & & 30,1 & \\
\hline & Solubilité protéines $\mathrm{KOH}(\%)$ & 89 & & 95 & & 91 & \\
\hline \multirow{7}{*}{$\begin{array}{l}\text { Extrudats } \\
\text { (sortie extrudeur) }\end{array}$} & Température fourreau $1\left({ }^{\circ} \mathrm{C}\right)$ & 87,0 & & 88,4 & & 87,3 & \\
\hline & Température fourreau $2\left({ }^{\circ} \mathrm{C}\right)$ & 153,8 & & 150, & & 152, & \\
\hline & Intensité (A) & $25,7-2$ & & 24,1 & & 25,7 & \\
\hline & Débit (kg/h) & 148 & & 151 & & 148 & \\
\hline & Humidité (\%) & 4,8 & & 6,6 & & 6,5 & \\
\hline & Solubilité protéines $\mathrm{KOH}(\%)$ & 80 & & 77 & & 78 & \\
\hline & Teneur en FAT (UTI/mg MSD) & 9,3 & & 8,1 & & 2,6 & \\
\hline \multirow{5}{*}{$\begin{array}{l}\text { Ecailles de pression } \\
\text { (sortie presse) }\end{array}$} & Sous-lot & $\mathrm{A} 1$ & $\mathrm{~A} 2$ & B1 & B2 & Z1 & $\mathrm{Z2}$ \\
\hline & Humidité (\%) & 6,7 & 5,5 & 8,0 & 7,4 & 7,2 & 7,7 \\
\hline & Teneur en huile (\% MS) & 5,9 & 5,3 & 6,2 & 5,6 & 5,3 & 6,0 \\
\hline & Solubilité protéines $\mathrm{KOH}(\%)$ & 79 & 78 & 73 & 79 & 76 & 80 \\
\hline & Teneur en FAT (UTI/mg MSD) & 10,4 & 10,2 & 3,9 & 4,8 & 3,2 & 3,2 \\
\hline
\end{tabular}

(1) MSD : matière sèche délipidée 
Tableau 5. Composition moyenne des tourteaux obtenus avec les deux procédés Aplatissage-Cuisson-Pression (ACP) (6 essais) et Extrusion-Pression (EP) (4 essais) à partir de graines des lots $A$ et $B$.

\begin{tabular}{|lllllll|}
\hline Caractéristiques des tourteaux & $\begin{array}{l}\text { Humidité } \\
\text { (\% brut) }\end{array}$ & $\begin{array}{l}\text { Huile résiduelle } \\
\text { (\% brut) }\end{array}$ & $\begin{array}{l}\text { Protéines } \\
\text { (\% brut) }\end{array}$ & $\begin{array}{l}\text { FAT } \\
\text { (UTI/mg brut) }\end{array}$ & $\begin{array}{l}\text { Solubilité } \\
\text { Protéines (\%) }\end{array}$ \\
\hline Graines Lot A & 11,0 & 18,8 & 35,3 & 33 & 89 \\
Graines Lot B & 12,7 & 19,8 & 33,9 & 38 & 95 \\
\hline ACP Tourteaux obtenus & Moyenne & $\mathbf{6 , 7}$ & $\mathbf{6 , 7}$ & $\mathbf{4 4 , 4}$ & $\mathbf{3 , 8}$ & 73 \\
\cline { 2 - 7 } à partir des lots A et B & mini-maxi & $5,3-7,9$ & $6,2-6,9$ & 42,5 à 45,8 & $3,1-4,6$ & $\mathbf{7 3}$ \\
\hline EP Tourteaux obtenus & Moyenne & $\mathbf{6 , 9}$ & $\mathbf{5 , 4}$ & $\mathbf{4 4 , 9}$ & $\mathbf{6 , 5}$ & $\mathbf{7 7}$ \\
à partir des lots A et B & mini-maxi & $5,5-8,0$ & $5,2-5,7$ & 43,8 à 46,4 & $3,4-9,1$ & $73-79$ \\
\hline
\end{tabular}

Les produits étant destinés à l'alimentation animale, leurs caractéristiques sont exprimées sur brut.

Tableau 6. Composition moyenne des tourteaux obtenus avec les deux procédés Aplatissage-Cuisson-Pression (ACP) (6 essais) et Extrusion-Pression (EP) (4 essais) à partir de graines des lots $A$ et $B$

\begin{tabular}{|c|c|c|c|c|c|c|}
\hline Procédé & Hypothèse & $\begin{array}{l}\text { Humidité } \\
\text { (\% brut) }\end{array}$ & $\begin{array}{l}\text { Huile résiduelle } \\
\text { (\% brut) }\end{array}$ & $\begin{array}{l}\text { Protéines } \\
\text { (\% brut) }\end{array}$ & $\begin{array}{l}\text { FAT } \\
\text { (UTI/mg brut) }\end{array}$ & $\begin{array}{l}\text { Solubilité } \\
\text { protéines (\%) }\end{array}$ \\
\hline \multirow{2}{*}{$\begin{array}{l}\text { Aplatissage-Cuisson } \\
\text {-Pression (ACP) }\end{array}$} & Optimiste & 4,5 & 6,4 & 44,9 & \multirow{2}{*}{$<8$} & \multirow{2}{*}{$>70 \%$} \\
\hline & Prudente & 4,5 & 7,2 & 44,5 & & \\
\hline \multirow[t]{2}{*}{ Extrusion-Pression (EP) } & Optimiste & 6,9 & 5,4 & 44,2 & \multirow[b]{2}{*}{$<12$} & \multirow[b]{2}{*}{$>75 \%$} \\
\hline & Prudente & 6,9 & 6,6 & 43,6 & & \\
\hline
\end{tabular}

Les produits étant destinés à l'alimentation animale, leurs caractéristiques sont exprimées sur brut.

des deux procédés (tableau 8 ) reste assez faible (de 1,4 à 6,0 €/tonne de graine triturée) si on le compare aux écarts qui peuvent exister entre les hypothèses de valorisation (plus de $60 € / \mathrm{t}$ ).

Les coûts de trituration sont pratiquement identiques pour les deux procédés (tableau 9) et s'élèvent environ à $33 € / t$ de graine triturée, les coûts de maintenance plus élevés pour le procédé EP étant compensés par une consommation énergétique plus faible. Dans le cadre des hypothèses étudiées et des cours des matières premières en 2011, la marge nette n'est positive que dans le cas d'une valorisation moyenne ou optimiste.

La comparaison des deux procédés doit également tenir compte de leur polyvalence pour la trituration d'autres grai- nes oléagineuses, ce qui peut s'avérer déterminant pour amortir les équipements en cas de contexte défavorable pour le soja. Le procédé ACP peut s'appliquer au soja ainsi qu'au colza et au tournesol alors que le procédé EP est réservé au soja.

\section{Conclusion}

Les travaux technologiques réalisés sur les deux procédés aplatissage-cuissonpression et extrusion-pression avaient pour objectif de faire le bilan de leurs forces et faiblesses pour équiper des unités industrielles de petite taille. Les essais réalisés à plusieurs échelles ont permis d'évaluer les effets des différents facteurs du traitement hydro-thermi- que, les caractéristiques des équipements, et de prévoir les niveaux de performances dans les conditions réelles de fonctionnement dans une unité industrielle. Ceux-ci sont proches mais néanmoins comportent des écarts (désactivation des facteurs antitrypsiques, déshuilage) qui peuvent motiver des choix différents pour un investissement dans un contexte donné.

Les coûts de trituration sont similaires et les vraies conséquences en matière économique se feront sur les possibilités de valorisation du tourteau et de I'huile. La valorisation locale de la graine de soja destinée à approvisionner les élevages de proximité est confrontée à la concurrence du tourteau de soja industriel importé. Ces tourteaux produits à bas

Tableau 7. Bilans matieres calculés à partir des performances des deux procédés Aplatissage-Cuisson-Pression (ACP) et Extrusion-Pression (EP) et des caractéristiques moyennes des graines de soja (1)

\begin{tabular}{|llll|}
\hline Bilans matière (pour 1000 kg de graines) & Tourteau $(\mathbf{k g})$ & Huile $(\mathbf{k g})$ & Eau perdue $(\mathbf{k g})$ \\
\hline EP - Rendement huile optimiste & 783,4 & 150,7 & 65,9 \\
\hline EP - Rendement huile prudent & 794,2 & 140,6 & 65,2 \\
\hline ACP - Rendement huile optimiste & 771,0 & 143,7 & 85,3 \\
\hline ACP - Rendement huile prudent & 778,0 & 137,0 & 85,0 \\
\hline
\end{tabular}

(1) Humidité $12 \%$ brut, MG : 19,4\% brut, protéines : 35,3\% brut (Feedbase, 2011). 
Tableau 8. Marges brutes (€/t de graine) pour les deux procédés Aplatissage-Cuisson-Pression (ACP) et Extrusion-Pression (EP), calculées selon trois hypothèses de valorisation

\begin{tabular}{|c|c|c|c|}
\hline & Valorisation optimiste & Valorisation moyenne & Valorisation pessimiste \\
\hline Tourteau (1) & Selon PROFAT (3) & Selon MAT (4) & Selon MAT (4) \\
\hline Huile (1) & $100 \%$ du cours & $100 \%$ du cours & $95 \%$ du cours \\
\hline Primes sur le tourteau (2) & $100 \%$ & $67 \%$ & $33 \%$ \\
\hline ACP - Rendement huile optimiste & 81,8 & 67,9 & 24,0 \\
\hline ACP - Rendement huile prudent & 80,8 & 66,8 & 18,2 \\
\hline EP - Rendement huile optimiste & 84,4 & 70,3 & 30,2 \\
\hline EP - Rendement huile prudent & 82,5 & 68,2 & 21,7 \\
\hline
\end{tabular}

(1) Valeur graines de soja : $350 € / \mathrm{t}$; huile : $910 € / \mathrm{t}$; tourteau soja $48: 311 € / \mathrm{t}$.

(2) Prime tourteau non OGM : $37,5 € / t$; prime traçabilité : $10 € / t$; prime proximité (économie transport) : $7 € / t$.

(3) Prix du tourteau déterminé par sa valeur PROFAT (teneurs huile + protéines) et par la valeur PROFAT et le prix du tourteau de soja 48.

(4) Prix du tourteau déterminé par sa teneur en MAT (protéines) et par la teneur en MAT et le prix du tourteau de soja 48.

Tableau 9. Comparaison des coûts de trituration avec les deux procédés Aplatissage-Cuisson-Pression (ACP) et Extrusion-Pression (EP) appliqués dans une unité traitant 20000 t de graines par an.

\begin{tabular}{|c|c|c|c|c|c|c|}
\hline \multirow[b]{2}{*}{ Coûts partiels } & \multicolumn{3}{|c|}{ Aplatissage-Cuisson-Pression (ACP) } & \multicolumn{3}{|c|}{ Extrusion-Pression (EP) } \\
\hline & $\begin{array}{l}\text { Base } \\
\text { de calcul }\end{array}$ & & $\begin{array}{l}\text { ( } € / \text { t de graine } \\
\text { triturée) }\end{array}$ & Base de calcul & & $\begin{array}{l}\text { ( } € / \text { t de graine } \\
\text { triturée) }\end{array}$ \\
\hline Broyeur & 37600 & $€$ & 0,27 & 37600 & $€$ & 0,27 \\
\hline Presse + concasseur + outils & 187420 & $€$ & 1,34 & 253017 & $€$ & 1,81 \\
\hline Cuiseur + distribution vapeur & 224580 & $€$ & 1,60 & 0 & $€$ & 0,00 \\
\hline Aplatisseur & 94000 & $€$ & 0,67 & 0 & $€$ & 0,00 \\
\hline Séparation des pieds & 16590 & $€$ & 0,12 & 16590 & $€$ & 0,12 \\
\hline Ensemble de filtration & 56421 & $€$ & 0,40 & 56421 & $€$ & 0,40 \\
\hline Extrusion & 0 & $€$ & 0,00 & 275000 & $€$ & 1,96 \\
\hline Refroidissement des écailles & 40560 & $€$ & 0,29 & 40560 & $€$ & 0,29 \\
\hline Manutentions & 120000 & $€$ & 0,86 & 120000 & $€$ & 0,86 \\
\hline Support charpentes & 16000 & $€$ & 0,11 & 16000 & $€$ & 0,11 \\
\hline Tuyauterie + calorifuge & 61500 & $€$ & 0,44 & 30750 & $€$ & 0,22 \\
\hline Armoires électriques + câbles & 132200 & $€$ & 0,94 & 132200 & $€$ & 0,94 \\
\hline Compresseur & 7000 & $€$ & 0,05 & 7000 & $€$ & 0,05 \\
\hline Frais montage & 33000 & $€$ & 0,24 & 30000 & $€$ & 0,21 \\
\hline Frais d'implantation LMM & 57630 & $€$ & 0,41 & 69156 & $€$ & 0,49 \\
\hline Total amortissement & 1084501 & $€$ & 7,75 & 1084294 & $€$ & 7,74 \\
\hline Frais financiers (1) & 22435 & $€ /$ an & 1,12 & 22431 & $€ /$ an & 1,12 \\
\hline Maintenance pression & 10008 & $€ /$ an & 0,50 & 13511 & $€ /$ an & 0,68 \\
\hline Maintenance reste équipements & 5044 & $€ /$ an & 0,25 & 4104 & $€ / a n$ & 0,21 \\
\hline Maintenance extrusion & 0 & $€ /$ an & 0,00 & 12375 & $€ /$ an & 0,62 \\
\hline Énergie thermique (2) & 150 & $\mathrm{kWh} / \mathrm{t}$ & 6,77 & 0 & $\mathrm{kWh} / \mathrm{t}$ & 0,00 \\
\hline Énergie électrique (2) & 72 & $\mathrm{kWh} / \mathrm{t}$ & 4,16 & 163 & $\mathrm{kWh} / \mathrm{t}$ & 9,41 \\
\hline Total énergie & & & 10,92 & & & 9,41 \\
\hline Total 1 & & & 20,28 & & & 19,51 \\
\hline Main d'œuvre & 210000 & $€ /$ an & 10,50 & 210000 & $€ /$ an & 10,50 \\
\hline Assurances & 7296 & $€ /$ an & 0,36 & 7296 & $€ /$ an & 0,36 \\
\hline BFCE (3) & 39981 & $€ / a n$ & 2,00 & 39981 & $€ / a n$ & 2,00 \\
\hline Total général & & & 33,14 & & & 32,37 \\
\hline
\end{tabular}

(1) amortissement 7 ans ; (2) prix vapeur : 0,045€/kWh, électricité : 0,05775€/kWh ; (3) besoin de financement du cycle d'exploitation 
coût dans de très grosses usines en Amérique du Sud ou aux États-Unis sont très compétitifs et une substitution par des tourteaux partiellement déshuilés ne sera possible que si les procédés sont correctement dimensionnés et optimisés. La valorisation des primes reste alors essentielle, pour garantir durablement une marge nette positive. Le débouché du tourteau vers des productions de qualité pour lesquelles la traçabilité et le caractère non-OGM sont recherchés est une condition de réussite ainsi que la mise en place d'une relation contractuelle équilibrée entre les divers opérateurs de la filière. Par ailleurs, la logistique devra aussi être optimisée pour réduire les coûts de transport. Enfin, le choix du procédé devra prendre en considération les risques d'une rupture de l'approvisionnement en soja et l'intérêt d'être polyvalent pour la trituration d'autres graines oléagineuses.

Remerciements. Les auteurs remercient François de la Perrière (Terres $d^{\prime}$ Alliance) et Philippe Lefèbvre (Coopérative Dauphinoise) pour la fourniture des graines et de données technico-économiques utilisées dans cette étude, ainsi qu'Hubert Hebinger, Didier Chollet et Louis-Marie Allard (CETIOM) pour leur implication dans le développement de ces travaux.
Conflits d'intérêts : aucun

\section{RÉFÉRENCES}

AOCS Official Method Ba 12-75. Determination of the total and residual trypsin inhibitors in food and feed products under the conditions of the test.

Feedbase. Composition graine de soja. Données économiques et techniques en alimentation animale. Cereopa-AFZ (www. feedbase.com), 2011.

Hereklman KL, Cromwell GL, Stahly TS, Knabe DA. Apparent digestibility of amino acids in raw conventional and low trypsin inhibitor soybeans for pigs. J Anom Sci 1992 ; 70 : 81826.

KeShun Liu. Soybean : Chemistry. In : Technology and utilization, Springer, 49, 1997.

Labalette F, Bourrel C, Jouffret P, Lecomte V, Quinsac A, Ledoux S. Panorama et futur de la filière du soja français. OCL 2010 ; 17 : 345-55.

Lareal. Les Méthodes de contrôle de la cuisson des sojas. 2009. Note technique $\mathrm{n}^{\circ} 73$ (https ://www.invivo-labs.com/module_externe/information_scientifique/document_73.pdf).

Melcion JP, Van der Poel AFB. Process technology and antinutritional factors : Principles, adequacy and process optimization. In : Van der Poel AFB, Huisman J, Saini HS (Eds), Recent advances of research in antinutritional factors in legume seeds. Wageningen Pers, Wageningen (Netherlands) : 419-34, 1993.

Oilword. In: Les marchés des oléagineux. Synthèse hebdomadaire $n^{\circ}$ 34-2012 du 16 au 23 août 2012. Cotations Rotterdam (http:// www.cetiom.fr/fileadmin/cetiom/Chiffres_ marches/lettres_de_marches/2012/34-2012. pdf), 2012.

Quinsac A, Bouvarel I, Buffo P, et al. L'extrusion-pression, procédé adapté à la trituration de graines de soja pour les filières avicoles locales tracées. In : Sixiemes Journées de la Recherche Avicole, St Malo: 292-6, 2005. http://www.journees-de-la-recherche.org/ index1024.php.

Rackis JJ, McGhee JE, Liener IE, Kakade ML, Puski G. Problems encountered in measuring trypsin inhibitor activity of soy flour. Report of a collaborative analysis. Cereal Science Today $1974 ; 19$ : 513-7.

Unip. Bilans des Matières Riches en Protéines, 2009/2010. Statistiques France et Europe. Unip, 2012. www.unip.fr.

Weder JKP, Kind A. Mode of action of the Kunitz trypsin and the Bowman-Birk proteinase inhibitors from soya against trypsins and chymotrypsins. In : Jansman AJM, Hill GD, Huisman J, van der Poel AFB (Eds.), Recent advances of research in antinutritional factors in legume seeds and rapeseed. Wageningen Pers, Wageningen: Netherlands: 61-4, 1998. 